\title{
گु \\ Observation of superradiant synchrotron radiation in the terahertz region
}

\author{
B. E. Billinghurst, ${ }^{*}$ J. C. Bergstrom, L. Dallin, M. de Jong, T. E. May, J. M. Vogt, and W. A. Wurtz \\ Canadian Light Source Inc., University of Saskatchewan, Saskatoon, Saskatchewan, S7N 2V3, Canada
}

(Received 1 May 2012; published 5 June 2013)

\begin{abstract}
We report the first high-resolution measurement of superradiance, using coherent synchrotron radiation in the terahertz region from the Canadian Light Source synchrotron and a Michelson interferometer with a nominal frequency resolution of $0.00096 \mathrm{~cm}^{-1}$. Superradiance arises when a high degree of phase coherence exists between the radiation fields of the individual electron bunches, and manifests itself as a series of narrow spectral peaks at harmonics of the bunch frequency. We observe an enhancement factor of 16 at the spectral peaks, limited by the interferometer resolution. The spectral distribution and relative amplitudes of the superradiant peaks are modified by altering the pattern of bunches along the bunch train.
\end{abstract}

PACS numbers: 41.60.Ap, 07.57.Hm

Electron synchrotrons and storage rings continue to evolve as important sources of synchrotron radiation across a wide spectral range with ever-improving beam qualities such as emittance and brightness. The radiation from such devices is usually incoherent due to the random phases of the electromagnetic fields emitted by the electrons. The electron beam in a synchrotron is segmented in the form of a bunch train and the incoherent radiation intensity per bunch is proportional to $N_{e}$, the number of electrons per bunch. On the other hand, coherent synchrotron radiation (CSR) is produced when the electrons emit in phase with each other. In principle, phase coherence obtains when the electrons are localized within a region small compared to the spectral wavelength of interest. When the electrons emit in phase, the associated fields add linearly and the spectral intensity per bunch varies as $\left(N_{e}\right)^{2}$. In practice, it is difficult to compress the bunch to the degree necessary to achieve complete coherence, so the number of electrons participating in CSR emission is generally a small fraction of the total number. Even so, the intensity gain over incoherent synchrotron radiation can easily exceed $10^{4}$ [1].

It has been understood for some time that when the electrons are bunched in a periodic sequence, the spectral intensity of the CSR is further enhanced in the form of intense narrow-bandwidth lines at harmonics of the bunch frequency [2]. In particular, the maximum intensity of these spectral lines is proportional to $\left(N_{b} N_{e}\right)^{2}$ while the line width varies as $1 / N_{b}$ where $N_{b}$ is the number of bunches in a given bunch train. This additional enhancement in the frequency domain due to bunch periodicity has been termed superradiance [3] as distinct from singlebunch coherence, and requires a high degree of phase

\footnotetext{
*Corresponding author. brant.billinghurst@lightsource.ca

Published by the American Physical Society under the terms of the Creative Commons Attribution 3.0 License. Further distribution of this work must maintain attribution to the author(s) and the published article's title, journal citation, and DOI.
}

coherence between the radiation fields of the individual bunches. Note that the radiation output of each electron is not altered in the transition to superradiance.

The earliest experiments on superradiance worked with Smith-Purcell (SP) radiation at terahertz frequencies $[4,5]$. SP radiation arises when an electron beam passes parallel to the surface of a periodic grating. The dispersion relation for SP radiation is $n \lambda=p[1 / \beta-\cos \theta]$ where $n$ and $\lambda$ are the order and wavelength, $\beta$ is the relativistic factor $v / c, \theta$ is the observation direction relative to the electron beam, and $p$ is the grating period. Thus, the wavelength can be tuned by altering the viewing angle. Theoretical studies of superradiant SP radiation continue, with the aim of developing a Smith-Purcell free electron laser [2,3].

Bunch-to-bunch coherence was first observed using the synchrotron radiation emitted by a bunch train from a linear accelerator and deflected by a magnet [6]. Coherence between adjacent bunches was confirmed using a low-resolution interferometer to observe the associated interferogram. A later study in a similar vein, but using coherent transition radiation, extended the coherence measurements to bunch pairs both adjacent and one bunch removed [7].

We report here the first high-resolution observation of superradiance using terahertz CSR from a synchrotron. The spectral resolving power used for this work greatly exceeds that of previous measurements of superradiant phenomena. We observe coherence between bunches that are separated by up to 14 bunches, using a high-resolution Michelson interferometer with an optical path-length difference (PLD) up to $9.4 \mathrm{~m}$. The bunch-to-bunch coherency manifests itself in the interferogram as a periodic sequence of patterns similar to the familiar autocorrelation (or center-burst) pattern. A Fourier transform of the interferogram to the frequency domain reveals the superradiant peaks at harmonics of the bunch frequency.

CSR was produced at the Canadian Light Source (CLS) in Saskatoon by operating the synchrotron at an energy of $1.5 \mathrm{GeV}$, with the momentum compaction adjusted to 
produce a bunch length of a few picoseconds. The CSR was produced in the continuous mode, where coherent radiation is continuously emitted by quasistable highfrequency components in the bunch charge distribution (see, for example, Ref. [8]). Details of the accelerator lattice properties typically used for the production of CSR at the CLS have been previously presented $[9,10]$. For these particular observations we measured synchrotron frequencies in the range 4.2 to $5.4 \mathrm{kHz}$, which correspond to momentum compaction factors of $3.5 \times 10^{-4}$ to $5.7 \times 10^{-4}$ and bunch lengths of 3.1 to $4.0 \mathrm{ps}$.

The onset of CSR in the far infrared region $\left(\sim 10 \mathrm{~cm}^{-1}\right)$ was evidenced by the rapid enhancement of the radiation flux, relative to the incoherent synchrotron radiation (ISR), as the bunch length was reduced at a fixed current. Flux amplifications of $\sim 5000$ were typical at the operational bunch length, and are characteristic of CSR production.

The $\mathrm{rf}$ frequency is $500 \mathrm{MHz}$ producing a bunch interval $T=2.0 \mathrm{~ns}$. The normal bunch train for these studies consists of 210 sequential bunches, out of a maximum of 285 . The initial current per bunch was approximately $23 \mu \mathrm{A}$, well below the threshold of the so-called bursting mode of CSR production in which the radiation from the bunches is emitted stochastically and thus is uncorrelated [11-13]. As expected, the superradiant effect disappeared when the bunch current was raised above the bursting threshold. The charge distribution among the bunches was uniform to within $\pm 10 \%$.

Spectra were acquired using a Bruker IFS 125 HR interferometer, using a $75 \mu \mathrm{m}$ Mylar beam splitter and an Infrared Laboratories Si Bolometer. A $10 \mathrm{~mm}$ aperture was used, with gain at $200 \times$, a scanner velocity of $\sim 25 \mathrm{~mm} / \mathrm{s}$ corresponding to an $\sim 80 \mathrm{kHz}$ sampling rate, and a resolution of $0.00096 \mathrm{~cm}^{-1}$ (defined as 0.9/PLD by Bruker convention). Each data set represents an average of between 12 and 32 scans. The CSR spectrum was measured in the far infrared region in the frequency range 6-15 $\mathrm{cm}^{-1}$, or approximately $0.2-0.5 \mathrm{THz}$.

Figure 1(a) shows the interferogram for the normal bunch train as a function of the optical PLD. For convenience, the fill pattern of the bunch train is denoted by the sequence $111111 \ldots$. The peak at the origin is the autocorrelation function, while the next large peak, at $\sim 600 \mathrm{~mm}$ PLD, represents the correlation between adjacent bunches due to their phase coherence. The final peak is the correlation of bunch-1 with bunch-16, bunch-2 with bunch-17, etc. In the future, we will refer to these features as bunch-to-bunch interference patterns. These patterns, with amplitudes comparable to the autocorrelation peak, are indicative of phase coherence, not only within each bunch, but also between bunches [6]. In other words, all electrons within the bunch train that contribute to the CSR at a given frequency are radiating in phase. Since this is the defining criterion for superradiance $[2,3]$, we argue that

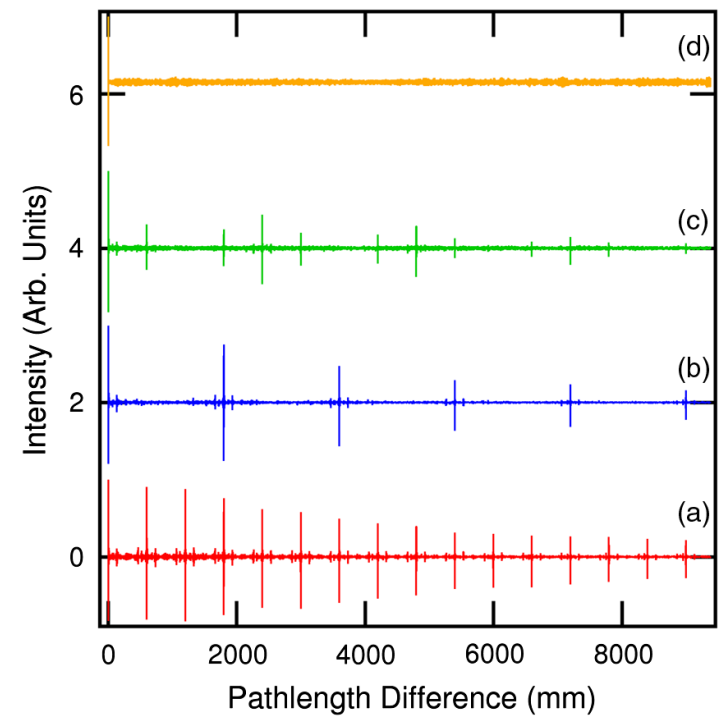

FIG. 1. Interferograms as a function of PLD for various bunchtrain fill patterns. The large peaks arise from the correlations between bunches separated along the train by a distance equal to the PLD. (a) Fill pattern $111111 \ldots$. ; (b) fill pattern $100100 .$. ; (c) fill pattern $11001100 \ldots$; (d) same as (a) but with incoherent synchrotron radiation [(b), (c), and (d) offset for clarity].

Fig. 1(a) is a manifestation of superradiance in the time domain. To support this argument, we note that measurements with ISR showed no discernible evidence of bunchto-bunch interference, as demonstrated in Fig. 1(d). Figure 2 shows an expanded view of the region around a path-length difference of $600 \mathrm{~mm}$, where the interference between adjacent bunches can be clearly observed in (a), whereas (d) shows no such interference. Similarly, bunchto-bunch coherence was suppressed when the CSR was generated in the bursting mode, which randomizes the radiation phases between bunches.

The attenuation of the individual interference patterns with increasing PLD is largely due to geometrical properties of the interferometer, and is common to both coherent

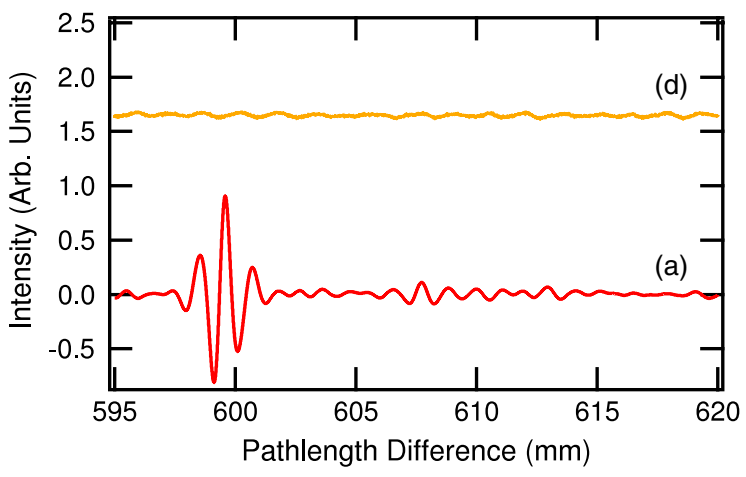

FIG. 2. Expanded view of Figs. 1(a) and 1(d) near a PLD of $600 \mathrm{~mm}$ representing the interference between adjacent bunches. Note that no evidence of interference between bunches is seen in $(d)$. 
and incoherent radiation. It might be argued that part of the attenuation derives from interbunch dynamics somehow causing a gradual phase change between bunches. Assuming such an effect would be a function of the bunch spacing, we altered the fill pattern by markedly increasing the bunch spacing. Specifically, pairs of bunches were removed between single bunches, so the train resembled the sequence $100100 \ldots$, tripling the bunch spacing. The results are displayed in Fig. 1(b) and are in excellent agreement with the corresponding bunch-to-bunch interference patterns in Fig. 1(a), suggesting that interbunch dynamics are not a significant issue here. Low-mode coupled-bunch synchrotron oscillations are not a factor either, due to their long wavelengths relative to the bunch train.

The strength of a given bunch-to-bunch interference pattern is proportional to the number of ways that $N_{b}$ bunches can contribute to a given overlay pattern. Thus, for a uniform sequence there are $N_{b}$ combinations for the autocorrelation peak, $N_{b}-1$ combinations for correlations between adjacent bunches, etc. This contributes to the downward slope in Fig. 1(a), but since $N_{b} \gg 1$, the effect is too small to be resolved. However, a simple change in the fill pattern can have a dramatic effect on the available combinations. To illustrate, in Fig. 1(c) we show the interferogram for the bunch pattern $11001100 \ldots$, for $N_{b}=106$. To the extent that $N_{b} \gg 1$, the expected sequence of peak amplitudes is proportional to $N_{b}, N_{b} / 2,0$, $N_{b} / 2, N_{b}, N_{b} / 2,0, N_{b} / 2$, etc. Allowing for the instrumental attenuations, the data indeed follow the expected pattern.

From rather general considerations, the interference patterns are described by the following expression:

$$
I(\tau)=\frac{1}{\pi} \int_{0}^{\infty}|f(\omega)|^{2}|g(\omega)|^{2}[1+\cos (\omega \tau)] d \omega,
$$

where

$$
|g(\omega)|^{2}=\left|\sum_{n=1}^{N_{b}} A_{n} \exp [i(n-1) \omega T]\right|^{2}
$$

Here $T$ is the time interval between bunches, $\tau$ is the PLD in the interferometer, and the constants $A_{n}$ describe the fill pattern of the bunch train. The quantity $|f(\omega)|^{2}$ is the CSR spectral intensity from a solitary bunch and is related to the intrinsic frequency spectrum of the bunch. For a uniform fill pattern $\left(A_{n}=1\right)$, Eq. (2) can be expressed in closed form:

$$
|g(\omega)|^{2}=\left[\frac{\sin \left(N_{b} \omega T / 2\right)}{\sin (\omega T / 2)}\right]^{2}
$$

This term expresses the multibunch interference [see Eq. (4.16) in Ref. [14] ]. It is strongly peaked at frequencies where the denominator vanishes, with a peak magnitude proportional to $N_{b}^{2}$ and a width proportional to $1 / N_{b}$. From Eq. (1), it samples narrow segments of the single-bunch spectrum at harmonics of the bunch frequency: $\omega_{n}=n \omega_{b}$ where $\omega_{b}=2 \pi / T$.

A Fourier transform of $I(\tau)$ to the frequency domain gives

$$
I(\omega)=|f(\omega)|^{2} \cdot|g(\omega)|^{2},
$$

which, for the uniform fill pattern, becomes

$$
I(\omega)=|f(\omega)|^{2} \cdot\left[\frac{\sin \left(N_{b} \omega T / 2\right)}{\sin (\omega T / 2)}\right]^{2} .
$$

This describes a sequence of superradiant peaks at harmonics of the bunch frequency as modulated by the singlebunch spectrum. The area of each peak, centered in an interval $\omega=\omega_{n} \pm \omega_{b} / 2$, is

$$
\int_{\omega_{n}-\omega_{b} / 2}^{\omega_{n}+\omega_{b} / 2} I(\omega) d \omega \approx N_{b}|f(\omega)|^{2} \omega_{b}
$$

neglecting the variation of the single-bunch spectrum across the interval. In other words, each highly localized superradiant peak contains the same number of photons as the multibunch CSR spectrum integrated across the interval $\omega_{b}$, in the absence of bunch-to-bunch coherence.

Let us clarify the meaning of $N_{b}$ in Eq. (5) when comparing with the experimental data, and we refer here to the peak width as limited by the instrumental resolution. The frequency resolution is directly related to the maximum PLD in the interferometer. The interferometer can accommodate up to 16 sequential bunches in a normal fill pattern (although it samples the entire bunch train) so the effective bunch number to be used in the shape function is $\bar{N}_{b}=16$, as distinct from $N_{b}$, the actual number of bunches in the train.

Figure 3 shows the entire frequency spectrum from the transform of Fig. 1(a) for the normal bunch sequence. The spectrum appears as a comb of superradiant peaks modulated by the single-bunch spectrum, which itself displays some structure. Also shown is a Fourier transform of the

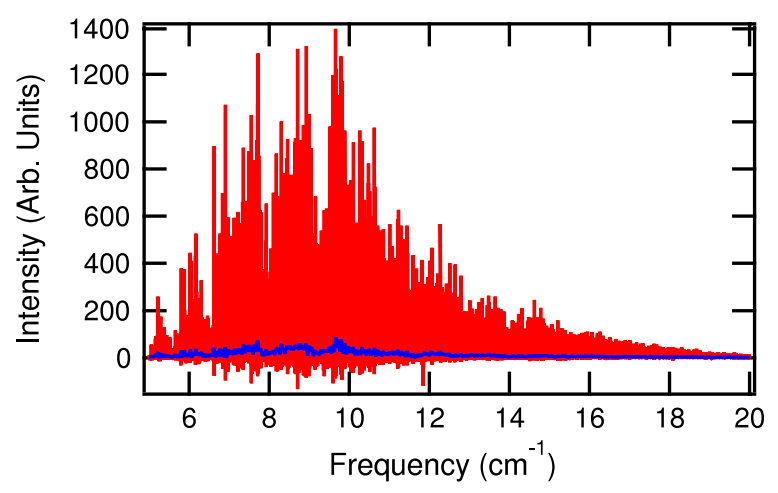

FIG. 3. Frequency spectrum for the normal bunch train showing the narrow superradiant peaks modulated by the singlebunch spectrum as described by Eq. (4) (red). Also shown is the low-resolution spectrum that precludes the observation of the effects of bunch-to-bunch coherence (blue) (see text). 
interferogram in Fig. 1(a) transformed at a nominal resolution of $0.05 \mathrm{~cm}^{-1}$; only the first $180 \mathrm{~mm}$ of PLD contribute to this transform and thus the effect of bunch-tobunch coherence is not observed. Therefore this spectrum is a good representation of the spectrum of 210 bunches in the absence of bunch-to-bunch coherence. For simplicity this will henceforth be referred to as the low-resolution spectrum. The comparison in Fig. 3 provides empirical evidence of the superradiant enhancement. The spectra are shown in finer detail in Fig. 4(a). A pronounced amplitude modulation is evident with a period of approximately $0.074 \mathrm{~cm}^{-1}$, which has its source in the small interference peaks seen in Fig. 1(a) bracketing the large peaks. These small peaks are a single-bunch phenomenon, and are currently under study.

Figure 4(b) shows a portion of the frequency spectrum derived from Fig. 1(b) corresponding to the fill pattern $100100 \ldots$... The closed-form expression Eq. (5) still applies except the bunch spacing has now tripled in size. From Eq. (5), the spacing between superradiant peaks in Fig. 4(b) should now be $1 / 3$ of that in Fig. 4(a), in agreement with the data.

Finally, Fig. 4(c) shows a portion of the spectrum derived from Fig. 1(c), corresponding to the fill pattern $11001100 \ldots$. The peaks are now arranged in groups of 3 , and the peak-peak spacing is $1 / 4$ that of Fig. 4(a). The side peaks of each grouping are half the height of the center peak, as predicted by Eq. (2).

Variations in the peak amplitudes between the three different fill patterns shown in Fig. 4(a)-4(c), can likely be attributed to differences in a number of parameters,

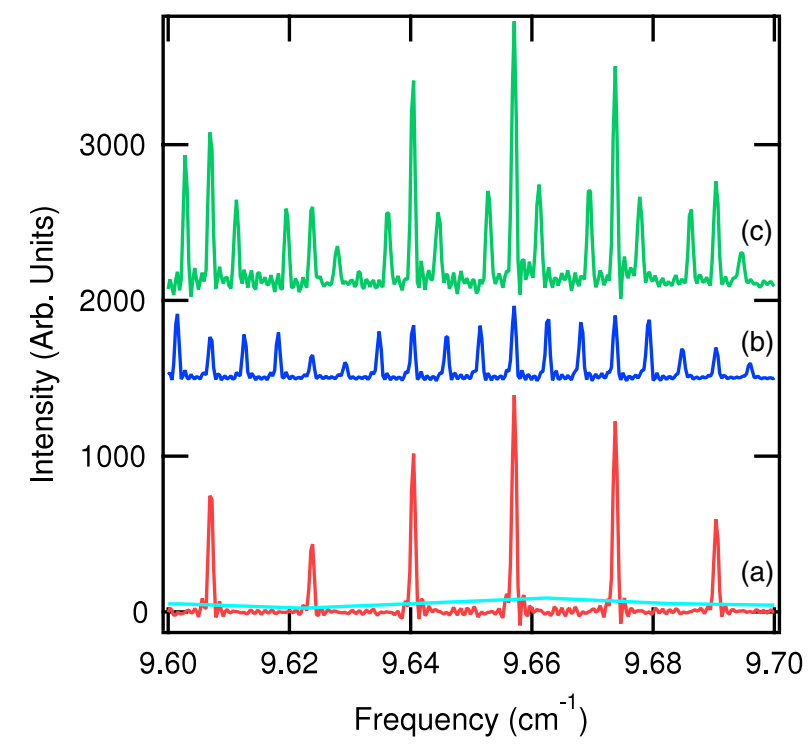

FIG. 4. The frequency spectra in detail showing the superradiant peaks for the various bunch-train fill patterns. (a) Fill pattern $11111 \ldots$ (low-resolution spectrum overlaid); (b) fill pattern $100100 \ldots$; (c) fill pattern $11001100 \ldots$ [(b) and (c) offset for clarity]. including current per bunch, degree of coherence of each bunch, degree of coherence between bunches, and variations in beam alignment. Unfortunately at this time our ability to monitor some of these parameters is insufficient to make any definite conclusions as to the effect of these parameters.

In Fig. 5 we show a few spectral lines from Fig. 4(a) in fine detail. Also shown is the line-shape based on the superradiant factor in Eq. (5), calculated for $\bar{N}_{b}=16$ as explained above, multiplied by the low-resolution spectrum, and normalized to the peak height. The measured peak shape is very well described by the model, even to the small diffraction like oscillations. The predicted enhancement factor at the peak is 16 relative to the multibunch CSR spectrum, as limited by the interferometer resolution. This is consistent with the peak enhancements as estimated empirically from Figs. 3 and 4. It is interesting to note that if the spectrometer had a PLD long enough to observe coherencies between bunches separated by 209 bunches, we would expect to see lines in the frequency spectrum that are a factor of 210/16 narrower and have maximum intensities 210/16 times higher than those observed in Fig. 4.

To summarize, we have observed coherent synchrotron radiation in the superradiant mode in the frequency range $6-15 \mathrm{~cm}^{-1}$ using a high-resolution interferometer. The necessary precondition is a high degree of phase coherence between the radiation fields along the bunch train. Enhancement factors of 16 relative to the multibunch CSR spectrum were achieved at the maxima of the superradiant peaks, limited by the resolution of the interferometer. This is in addition to true amplifications of $10^{3}-10^{4} \mathrm{per}$ bunch in the transition from incoherent to coherent synchrotron radiation. The frequency distribution and relative amplitudes of the spectral peaks can be modified by altering the fill pattern of the bunch train, as demonstrated for three different patterns. Thus, all measurements reported

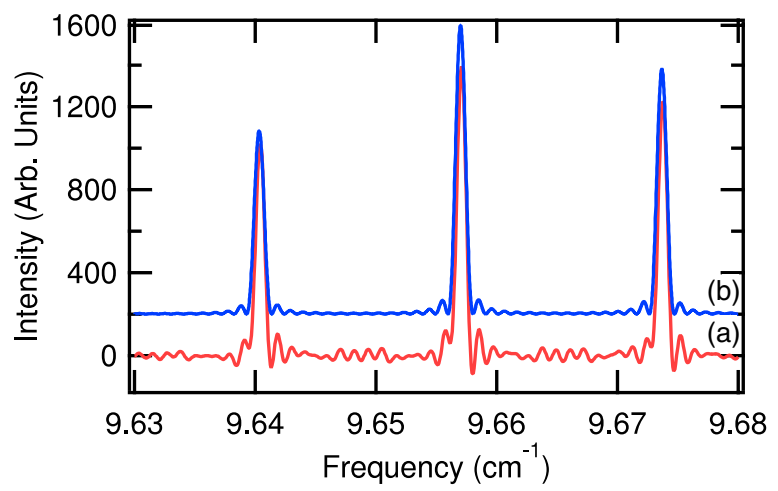

FIG. 5. (a) Spectral peaks from Fig. 4(a) in fine detail. (b) The line-shape based on the superradiant factor in Eq. (5), calculated for $\bar{N}_{b}=16$ as explained in the text and multiplied by the lowresolution spectrum and normalized to the peak height. The predicted peak width $1 /\left(T \bar{N}_{b}\right) \approx 0.0010 \mathrm{~cm}^{-1}$ agrees well with the data (offset for clarity). 
here are consistent with a simple description of superradiance arising from bunch-to-bunch coherence in the terahertz region. These observations demonstrate the potential for significant advancement in terahertz spectroscopy, particularly if a technique for tuning the frequency of the observed spectral peaks can be developed.

\section{ACKNOWLEDGMENTS}

The authors would like to acknowledge the support of Dr. Thomas Ellis and Elder Matias. Research described in this paper was performed at the Canadian Light Source, which is supported by the Natural Sciences and Engineering Research Council of Canada, the National Research Council Canada, the Canadian Institutes of Health Research, the Province of Saskatchewan, Western Economic Diversification Canada, and the University of Saskatchewan.

[1] M. Abo-Bakr, J. Feikes, K. Holldack, P. Kuske, W. B. Peatman, U. Schade, G. Wüstefeld, and H.-W. Hübers, Phys. Rev. Lett. 90, 094801 (2003).

[2] A. Gover, Phys. Rev. ST Accel. Beams 8, 030701 (2005).

[3] H. L. Andrews, C.H. Boulware, C. A. Brau, and J.D. Jarvis, Phys. Rev. ST Accel. Beams 8, 110702 (2005).
[4] J. Urata, M. Goldstein, M.F. Kimmitt, A. Naumov, C. Platt, and J.E. Walsh, Phys. Rev. Lett. 80, 516 (1998).

[5] S. E. Korbly, A. S. Kesar, J. R. Sirigiri, and R. J. Temkin, Phys. Rev. Lett. 94, 054803 (2005).

[6] Y. Shibata, T. Takahashi, K. Ishi, F. Arai, H. Mishiro, T. Ohsaka, M. Ikezawa, Y. Kondo, S. Urasawa, T. Nakazato, R. Kato, S. Niwano, and M. Oyamada, Phys. Rev. A 44, R3445 (1991).

[7] T. Takahashi, T. Matsuyama, K. Kobayashi, Y. Fujita, Y. Shibata, K. Ishi, and M. Ikezawa, Rev. Sci. Instrum. 69, 3770 (1998).

[8] F. Sannibale, J. M. Byrd, A. Loftsdóttir, M. Venturini, M. Abo-Bakr, J. Feikes, K. Holldack, P. Kuske, G. Wüstefeld, H.-W. Hübers, and R. Warnock, Phys. Rev. Lett. 93, 094801 (2004).

[9] L. O. Dallin and M. S. de Jong, in Proceedings of the 23rd Particle Accelerator Conference, Vancouver, Canada (IEEE, Piscataway, NJ, 2009), p. 1126.

[10] W. A. Wurtz and L. O. Dallin, in Proceedings of First International Particle Accelerator Conference, Kyoto, Japan (2010), p. 2484.

[11] G. Stupakov and S. Heifets, Phys. Rev. ST Accel. Beams 5, 054402 (2002).

[12] M. Venturini and R. Warnock, Phys. Rev. Lett. 89, 224802 (2002).

[13] M. Venturini, R. Warnock, R. Ruth, and J. A. Ellison, Phys. Rev. ST Accel. Beams 8, 014202 (2005).

[14] D. J. Wingham, Phys. Rev. D 35, 2584 (1987). 